\title{
ESR Studies of Phosphorescent Corannulene; Evidence for Pseudorotation*
}

\author{
Fitzgerald B. Bramwell \\ Department of Chemistry, University of Michigan, Ann Arbor, Michigan 48104 \\ AND \\ JULien Gendell \\ Department of Chemistry, Oakland University, Rochester, Michigan 48063
}

(Received 21 January 1970)

\begin{abstract}
Electron spin resonance (ESR) spectra have been recorded for the lowest triplet state of corannulene. The experiments were performed by ultraviolet irradiation of the molecule in rigid glass solutions at 77 and $15^{\circ} \mathrm{K}$. In the high-field $(\Delta m=1)$ region the line shape and line positions of the absorptions associated with the $X$ and $Y$ magnetic axes were strongly temperature dependent. From an analysis of the spectra the following conclusions were drawn: Corannulene in its first triplet state has a configuration of lowest energy which is not fivefold symmetric; at $15^{\circ} \mathrm{K}$ corannulene gives in the high-field region the spectrum of a molecule "frozen" in conformations of less than threefold symmetry; tunneling between equivalent conformations at $77^{\circ} \mathrm{K}$ is believed to occur at a rate of about $10^{9} \mathrm{~Hz}$.
\end{abstract}

\section{INTRODUCTION}

The observation of electron spin resonance spectra of the triplet state for both mixed crystals and rigid glass solutions of aromatic and partially aromatic compounds has been of considerable interest in the last several years. Spectra have been observed of many molecules which may be described as orbitally degenerate or near degenerate. In these systems theoretical treatments have used first- and second-order dynamical and static Jahn-Teller effects as a guide to the nature of intramolecular interactions. ${ }^{1}$ The reduced symmetry observed in the spectra of phosphorescent cations, ${ }^{2}$ which are characterized by a threefold or higher symmetry axis in the un-ionized ground state, have also been interpreted in terms of static Jahn-Teller effects.

Temperature-dependent spectra for phosphorescent systems have been interpreted as indicative of a dynamic process. ${ }^{3}$ For molecules such as triptycene and tribenzotriptycene, which may be characterized as having aromatic subsystems linked by a $\sigma$-bonded carbon frame, such spectra have been interpreted in terms of a model in which a transfer of spin polarization between subsystems is postulated. For "totally" conjugated molecules such as benzene and mesitylene, temperature-dependent spectra have been interpreted in terms of a dynamical interconversion between three or more Jahn-Teller distorted molecular configurations of less than threefold symmetry. For benzene, a small temperature-dependent shift of the $H_{\min } \operatorname{line}^{3}$ and a spectrum $^{4}$ at $2^{\circ} \mathrm{K}$ characteristic of a distorted conformation was considered to be evidence for this type of motion. For mesitylene, a temperature-dependent shift without line broadening of the absorption lines was recorded of the $\Delta m=1$ region of the triplet spectrum of the mixed crystal. ${ }^{5}$

We should like to report the observation of temperature-dependent $\Delta m=1 \mathrm{ESR}$ spectra for a unique and new member of this class of orbitally degenerate aromatic molecules, corannulene. Corannulene ${ }^{6}$ is a highly strained aromatic hydrocarbon whose ground-state conformation is described by the symmetry operations of the $C_{5 v}$ point group (see Fig. 1). X-ray studies indicate that the atoms which lie on the molecular perimeter are in a plane $1 \AA$ above the pentagonal inner ring, creating a bowllike structure in the ground state. ${ }^{7}$ The NMR proton spectrum yields a single peak at $\tau=+2.19 .^{6}$ ESR studies of the radical anion have

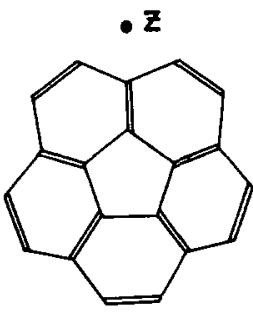

(A)

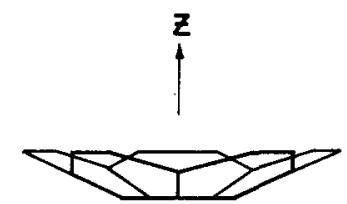

(B)
Fig. 1. Ground-state configuration of corannulene: (A) planar projection; (B) stereoscopic projection.

indicated that a fivefold symmetry is preserved at room temperature and that the molecule is aromatic although it is nonplanar and nonalternant. ${ }^{8}$

\section{EXPERIMENTAL}

A $10^{-3} \mathrm{M}$ solution of corannulene in dimethylformamide (DMF) and ethanol (EtOH) (1:4 by volume) was prepared in high-quality quartz tubing of $2 \mathrm{~mm}$ i.d. The sample was thoroughly degassed.

The ESR measurements were conducted on a Varian $\mathrm{V} 4502 \mathrm{X}$ band spectrometer with $100 \mathrm{KHz}$ modulation with the rf perpendicularly polarized to the static field. Absolute magnetic field strengths were measured by a proton magnetic resonance field meter, similar in design to that described by Ingram, ${ }^{9}$ used in conjunction with a Hewlet-Packard 5245L frequency counter.

Measurements at temperatures of 77 and $15^{\circ} \mathrm{K}$ were performed in a modified Varian variable temperature 
Dewar, the sample being cooled by the flow of helium or nitrogen gas from vaporized liquid through the Dewar. At $15^{\circ} \mathrm{K}$ the temperature was determined by measuring the change in resistance of a calibrated carbon resistor. At $77^{\circ} \mathrm{K}$ the temperature was measured by the use of a copper-constantan thermocouple. Ultraviolet radiation from an Osram $1000 \mathrm{~W}$ HBO lamp was focused upon the irradiation slits of the cavity by a quartz lens. Phosphorescent lifetimes were estimated by shuttering the uv excitation and recording the decay of the resonance signal.

Because of the limited supply of corannulene it was impossible to perform accurate experiments in other matrices or at other temperatures. The one available sample was reused in a 2-methyltetrahydrofuran (2-MeTHF) solution after data had been obtained from the DMF: EtOH solvent system.

As an independent check on the accuracy of our measurements the spectrum of phosphorescent coronene in DMF: EtOH was obtained. Quantitative agreement was found with the results reported by Lhoste et $a l .{ }^{10} \mathrm{~A}$ uv spectrum $(3000-4000 \AA)$ of the solution taken on a Cary 14 at room temperature both before and after irradiation did not indicate that any photolytic decomposition of the corannulene had occurred.

A FORTRAN IV program to simulate the observed spectra was written in the same style as that reported by Wasserman et al., ${ }^{11}$ differing, however, in several important aspects: (1) The program simulates the spectrum within $\pm 50 \mathrm{G}$ of a stationary resonance field; (2) the spectrum of the $H_{\min }$ resonance is not calculated; (3) the program has incorporated an option to apply Boltzmann statistics so that line shapes and spectral intensities, provided no dynamical processes are occurring, may be calculated as a function of temperature, particularly for the case when $h \nu \cong k T$; (4) there is an option to simulate either the entire $\Delta m=1$ spectrum or only that region of the spectrum extending below $g=2$; (5) an option for a Gaussian or Lorentzian line shape which is calculated for five or 10 linewidths with the line center at the stationary resonance field is available.

An IBM 360/67 computer requires anywhere from 68 to $167 \mathrm{sec}$ to simulate a spectrum, depending on which options are used.

\section{RESULTS}

The experimental spectra at 77 and $15^{\circ} \mathrm{K}$ and computer simulations are shown in Figs. 2 and 3. Both experimental spectra show a strong absorption in the $g=2$ region at about $3300 \mathrm{G}$. The signal in this region is due to free radicals produced by the photolytic decomposition of the solvent. Since the intensity of this free radical signal is large, it will hide any weaker triplet transitions which may occur at nearby resonance fields. All features of the spectra were checked for their light dependence by observing whether or not the feature remained or decayed within a few seconds after the

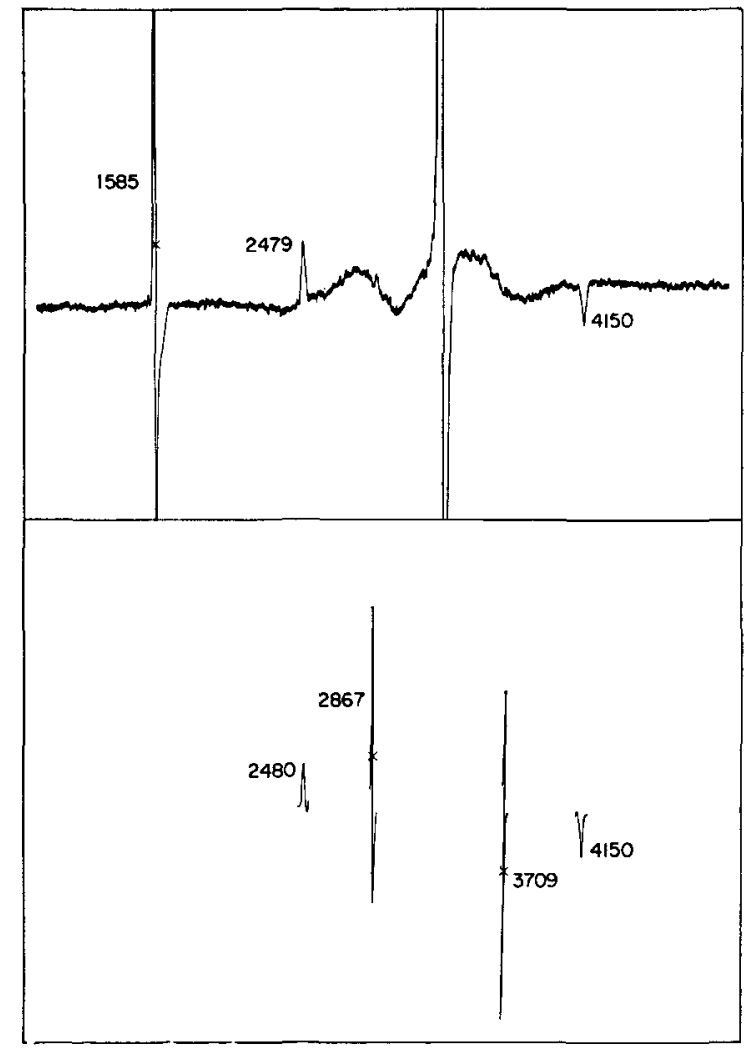

FIG. 2. Experimental and computed spectra at $77^{\circ} \mathrm{K}$. Top: experimental spectrum; $\delta=0.3105 \mathrm{~cm}^{-1}, X=Y=0.0261 \mathrm{~cm}^{-1}$, $Z=-0.0522 \mathrm{~cm}^{-1}$. The large signal in the experimental spectrum near $3300 \mathrm{G}$ is due to a photolytically produced free radical. Bottom: computer-simulated spectrum for $\Delta m=1$ region.

uv excitation was removed. The phosphorescent lifetime measured at $77^{\circ} \mathrm{K}$ was $2-3 \mathrm{sec}$ and at $15^{\circ} \mathrm{K}$ was $4-5 \mathrm{sec}$.

The experimental spectra are interpreted in terms of a spin Hamiltonian which considers only Zeeman and dipolar couplings, other effects being orders of magnitude smaller, ${ }^{12}$ and is given by

$$
\begin{gathered}
\mathfrak{H}=\mathfrak{H}_{z}+\mathfrak{H}_{D}=g \beta H_{0} \cdot S-X S_{x}{ }^{2}-Y S_{y}{ }^{2}-Z S_{z}{ }^{2}, \\
X+Y+Z=0
\end{gathered}
$$

or

$$
\begin{gathered}
\mathfrak{K}_{D}=D\left(S_{z}{ }^{2}-1 / 3 S^{2}\right)+E\left(S_{x}{ }^{2}-S_{y}{ }^{2}\right), \\
D=1 / 2(X+Y)-Z, \\
E=-1 / 2(X-Y) .
\end{gathered}
$$

$X, Y$, and $Z$ are the principal values of the traceless zero field splitting tensor and can be represented in terms of two parameters $D$ and $E$, the zero field energies.

For perpendicularly polarized oscillating magnetic fields the first derivative spectrum for randomly oriented triplets should exhibit six features in the $\Delta m=1$ region corresponding to a large change of absorption at these stationary resonance field values. Two stationary resonance fields are associated with each of the three magnetic axes of the molecule $(X, Y$, or $Z)$. The 
TABLE I. Observed and computed resonance fields in gauss.

\begin{tabular}{|c|c|c|c|c|c|c|c|c|}
\hline & $T\left({ }^{\circ} \mathrm{K}\right)$ & $H_{\mathrm{min}}$ & $H z 3$ & $I I y 2$ & $11 x^{2}$ & $H x 3$ & $I_{y 3}$ & $H z 2^{\mathrm{c}}$ \\
\hline Experimentala & 77 & $1585 \pm 4$ & $2479 \pm 4$ & e & $\mathrm{e}$ & $\mathbf{e}$ & $\mathrm{e}$ & $4150 \pm 4$ \\
\hline Computedf & & 1586 & 2480 & 2867 & 2867 & 3709 & 3709 & 4150 \\
\hline Experimental ${ }^{\mathrm{b}}$ & 15 & $1564 \pm 4$ & $2438 \pm 4$ & $2568 \pm 4$ & $\mathrm{~d}$ & d & $3984 \pm 4$ & $4145 \pm 4$ \\
\hline Computedg & & 1561 & 2436 & 2569 & 3103 & 3404 & 3985 & 4143 \\
\hline
\end{tabular}

a Line positions determined from spectra as described in text $\delta=$ $0.3105 \mathrm{~cm}^{-1}$.

${ }^{b}$ Line positions determined from spectra as described in text $\delta=$ $0.3080 \mathrm{~cm}^{-1}$.

${ }^{c}$ For line designations, see text, Figs. 2 and 3.

d Absorption obscured by free radical signal.

magnetic field values of these six features are denoted $H x 2, H x 3, H y 2, \cdots$, and are determined by the values of $X, Y, Z$ (or $D$ and $E$ ) according to equations ${ }^{13}$

$$
\begin{aligned}
& H x 2=(2 g \beta)^{-1}\left[(2 \delta-3|X|)^{2}-(Y-Z)^{2}\right]^{1 / 2}, \\
& H \times 3=(2 g \beta)^{-1}\left[(2 \delta+3|X|)^{2}-(Y-Z)^{2}\right]^{1 / 2} .
\end{aligned}
$$

The stationary resonance fields for the $Y$ and $Z$ directions are obtained through cyclic permutations of Eqs. (5) and (6).

In addition, there is another feature at low fields labeled $H_{\mathrm{min}}$, whose value is given by the following equation $^{13}$

$$
H_{\min }=(2 g \beta)^{-1}\left[\delta^{2}+4(X Y+X Z+X Z)\right]^{1 / 2} .
$$

$\delta$ is defined as the microwave energy, and $g$ and $\beta$ have their usual meanings. In this manner, seven features in the first derivative spectrum are determined by two molecular parameters $D$ and $E$.

In the case of a triplet with threefold or higher symmetry $X=Y(E=0)$. The $X$ and $Y$ axes are magnetically equivalent, and four $\Delta m=1$ and one $H_{\min }$ absorption are predicted. Thus, five experimental field values are determined by the value of the one molecular parameter $D$.

Stationary resonance field values were determined from the experimental spectra in the same manner as reported by Lhoste et $a l .{ }^{10}$ and are given in Table I. From these resonance field values, $D$ and $E$ were obtained by a least-squares analysis using Eqs. (5) - (7) . Computed values for the stationary resonance fields using the least-squares values of $D$ and $E$ are reported in Table I. Since the signs of the zero field splittings can not be determined by these methods except through extensive studies at liquid-He temperature, ${ }^{14} Z$ was arbitrarily chosen less than zero. In addition, the convention $|Z|>|Y|>|X|$ was adopted. ${ }^{13}$

Figure 2 shows the spectrum observed at $77^{\circ} \mathrm{K}$. By assigning the two narrow peaks observed in the $\Delta m=1$ region to the $Z$ magnetic axis and through location of the $H_{\text {min }}$ peak, values of $D=0.0783 \pm 0.0003 \mathrm{~cm}^{-1}$ e Approximate centers of broadened signal agree with computed values.

f As described in text $D=0.0783 \mathrm{~cm}^{-1} \pm 0.0003 \mathrm{~cm}^{-1} ; E=0.00 \pm 0.0003$ $\mathrm{cm}^{-1} ; g=2.0060$.

$\mathrm{g}$ As described in text $D=0.0796 \mathrm{~cm}^{-1} \pm 0.0003 \mathrm{~cm}^{-1} ; E=0.0173 \pm 0.0003$ $\mathrm{cm}^{-1} ; g=2.0023$.

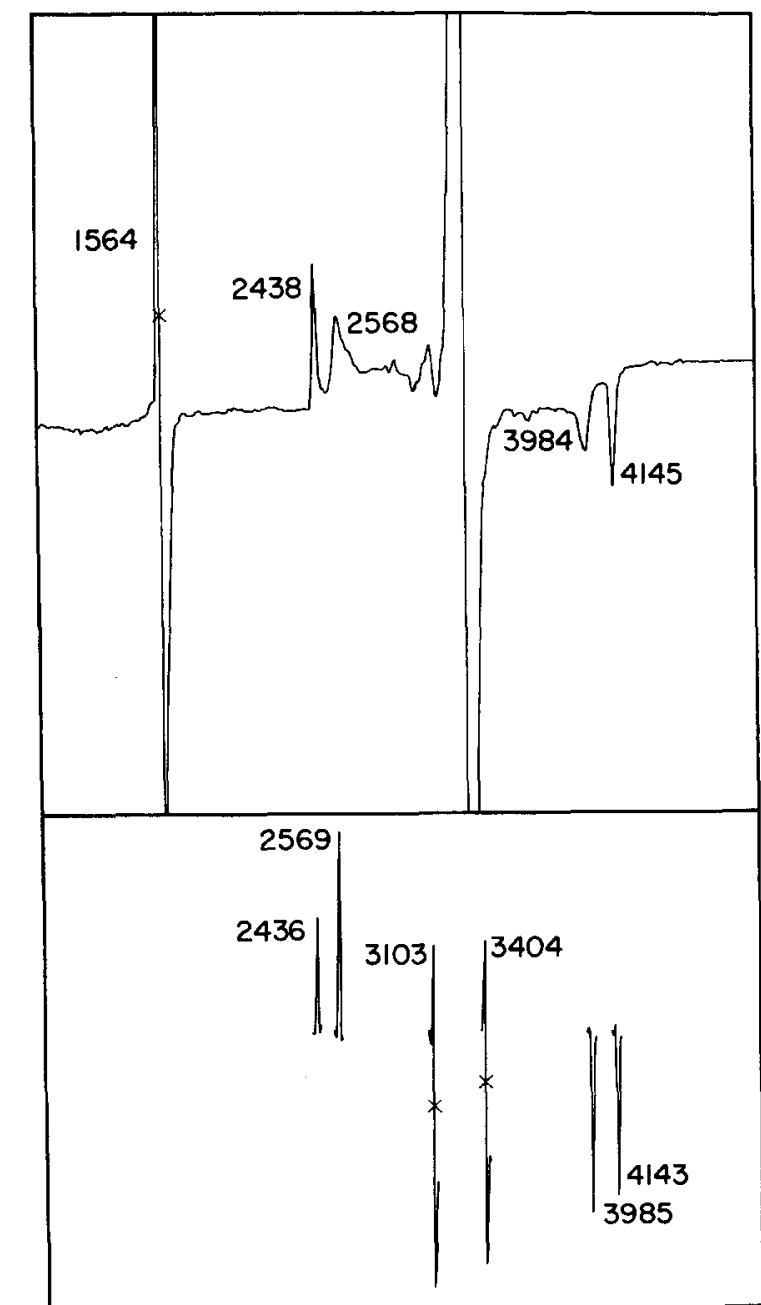

FIG. 3. Experimental and computed spectra at $15^{\circ} \mathrm{K}$. Top: experimental spectrum; $\delta=0.3080 \mathrm{~cm}^{-1}, Y=0.0439 \mathrm{~cm}^{-1}, X=$ $0.0092 \mathrm{~cm}^{-1}, Z=-0.0531 \mathrm{~cm}^{-1}, g=2.0023$. The large signal in the experimental spectrum near $3300 \mathrm{G}$ is due to a photolytically produced free radical. Bottom: computer-simulated spectrum for $\Delta m=1$ region. 
and $E=0.0 \pm 0.003 \mathrm{~cm}^{-1}$ were obtained. The value of $g$ was varied in the least-squares analysis, and the best agreement (which is well within the experimental uncertainty) between the experimental and computed stationary field values was obtained with $g=2.0060$. The changes in the stationary resonance fields due to this change in $g$ from the free electron value are small amounting to only 3 or $4 \mathrm{G}$. The experimental spectrum did not exhibit a derivative line shape characteristic of molecules with degenerate $X, Y$ magnetic axes and $E=0$ at the calculated fields of 2867 and $3709 \mathrm{G}$. Instead, a quite broadened signal whose approximate center position is in the region of these calculated fields was observed.

The spectrum observed at $15^{\circ} \mathrm{K}$ is shown in Fig. 3 . The free radical absorption appears to have some structure on it, including an apparent peak at about $3050 \mathrm{G}$. These features of the spectrum are not light sensitive, however, and remain after irradiation has been discontinued.

The most noticeable change in the spectrum at $15^{\circ} \mathrm{K}$ from that at $77^{\circ} \mathrm{K}$ spectrum is the presence of two new narrow peaks. An analysis of the $15^{\circ} \mathrm{K}$ spectrum which assigns the additional peak to the $Y$ magnetic axes yields the result $D=0.0796 \pm 0.0003 \mathrm{~cm}^{-1}$, $E=0.0173 \pm 0.0003 \mathrm{~cm}^{-1}$, and $g=2.0023$. There is excellent agreement between the experimental and computed field values (see Table I). The features associated with the $X$ magnetic axis were not observed, and our analysis indicates that they are hidden by the large $g=2$ free radical absorption (see Table I and Fig. 3).

The results obtained in the 2-MeTHF matrix at $20^{\circ} \mathrm{K}$ were qualitatively similar to those in DMF: EtOH at $77^{\circ} \mathrm{K}$. The features associated with the $X, Y$ magnetic axes were considerably narrower in 2-MeTHF at $20^{\circ} \mathrm{K}$ than in DMF-EtOH at $77^{\circ} \mathrm{K}$, but no additional peaks that could be associated with the $Y$ magnetic axis were observed and the observed features were consistent with the value $E=0$.

\section{DISCUSSION}

The resolution in DMF-EtOH at $15^{\circ} \mathrm{K}$ of the broadened signals observed at $77^{\circ} \mathrm{K}$ and the nonzero value of $E$ at $15^{\circ} \mathrm{K}$ indicates that there is a distortion at $15^{\circ} \mathrm{K}$ which is not seen at $77^{\circ} \mathrm{K}$ and that at $15^{\circ} \mathrm{K}$ corannulene in its first excited triplet state has less than threefold symmetry.

The broadened signals observed in the spectrum at $77^{\circ} \mathrm{K}$ could be explained in terms of a variable site distortion with the same values of $Z$ for each site but a distribution of $X$ and $Y$ values such that on the average $X=Y$ and $E=0$, or in terms of a broadening process caused by intramolecular motion. A variable site distortion would reflect minor distortions of corannulene in the matrix but would preserve the over-all groundstate molecular symmetry. It is probable that line broadening due to a variable site distortion would increase as the temperature is decreased.

In contrast, reduced intramolecular motion, at lower temperatures, would narrow the broad lines associated with the $X, Y$ magnetic axes, and cause them to split into four features of narrower linewidth if the triplet state of corannulene was distorted at low temperatures such that $E \neq 0$. The features of the experimental spectrum at $15^{\circ} \mathrm{K}$ and the analysis of this spectrum which gives $E \neq 0$ are in complete agreement with this latter explanation, indicating that the observed broadening at $77^{\circ} \mathrm{K}$ is due to some form of intramolecular motion.

To understand the type of motion that may be expected to occur we invoke a model similar to that proposed by van der Waals and de Groot. ${ }^{3}$ Corannulene in a distorted conformation is allowed to rotate only about its $Z$ axis (see Fig. 1). If the rotation is fast, the $X$ and $Y$ axes will appear degenerate and the observed spectrum will be characterized by $E=0$. The rate of rotation for the artificial degeneracy to set in can be approximated as being greater than

$$
\begin{aligned}
\Delta \omega_{x y} & \cong(g \beta / \hbar)\left|H_{x}-H_{y}\right| \\
& \cong 9 \mathrm{GHz} .
\end{aligned}
$$

As the rotation slows down with lowered temperature, it will be possible to resolve $X$ and $Y$ separately and a spectrum in which $E \neq 0$ will be observed. In the intermediate region between fast and slow rotation a broad spectrum characteristic of an unresolved distribution of the $X, Y$ resonance fields will be observed. Since the rotation occurs about the $Z$ axis, any change in the value of the $Z$ zero field splitting is expected to be small, and this is what is observed experimentally. This model also predicts an increase in $|E|$ on decreasing the temperature from 77 to $15^{\circ} \mathrm{K}$. An increase in $E$ with a small change in $Z$ would cause the position of the $H_{\min }$ line to shift to lower fields, and this is also observed to occur experimentally (see Table II).

In general, for a randomly oriented sample the zero field splittings axes $(X, Y, Z)$ can not be experimentally assigned to the molecular axes, $(x, y, z)$. For corannulene, however, the small change in $Z$ with temperature and the value $E=0$ at $77^{\circ} \mathrm{K}$ indicate that the $Z$ zero field splitting must be associated with the molecular pseudo-fivefold symmetry axis $z$.

The motion described here is hypothetical since the large frequency of rotation, the low temperatures, and large molecular size of corannulene are inconsistent with a free rotation about the $Z$ axis and little or no rotation about the $X$ and $Y$ axes. However, a pseudorotational mechanism would also explain the experimental results. A pseudorotation would involve minor nuclear displacements with the molecule tunneling between equivalent conformers which have less than threefold symmetry. Thus, pseudorotation gives the appearance of a free rotation without the necessity of 
TABLE II. Stationary resonance fields (corrected to $\delta=0.3080 \mathrm{~cm}^{-1}$ ).

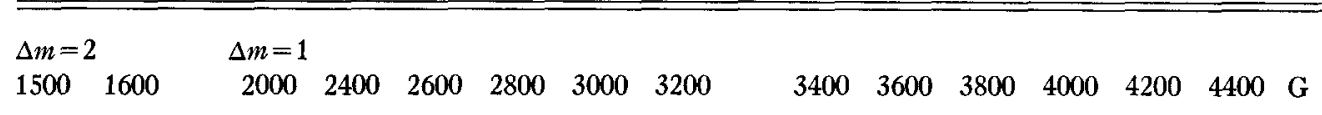

A. Position of stationary fields for corannulene in DMF:EtOH(1:4). Model exhibits characteristics of fast "rotation" about $z$ axis.

\begin{tabular}{|c|c|c|c|c|c|}
\hline 1572 & 2453 & 2840 & 3683 & 4125 & $X=Y=0.0261 \mathrm{~cm}^{-1}$ \\
\hline 1 & 1 & 1 & 1 & I & $Z=-0.0522 \mathrm{~cm}^{-1}$ \\
\hline$H_{\min }$ & $H_{2}$ & $H_{x, y^{\mathrm{a}}}$ & $H_{x, y^{\mathbf{8}}}$ & $H_{z}$ & $\begin{aligned} D & =0.0783 \mathrm{~cm}^{-1} \\
E & =0.00 \mathrm{~cm}^{-1} \\
T & =77^{\circ} \mathrm{K} \\
g & =2.0060\end{aligned}$ \\
\hline
\end{tabular}

B. Position of stationary fields for corannulene in DMF:EtOH(1:4). Model exhibits characteristics of slow/static configuration.

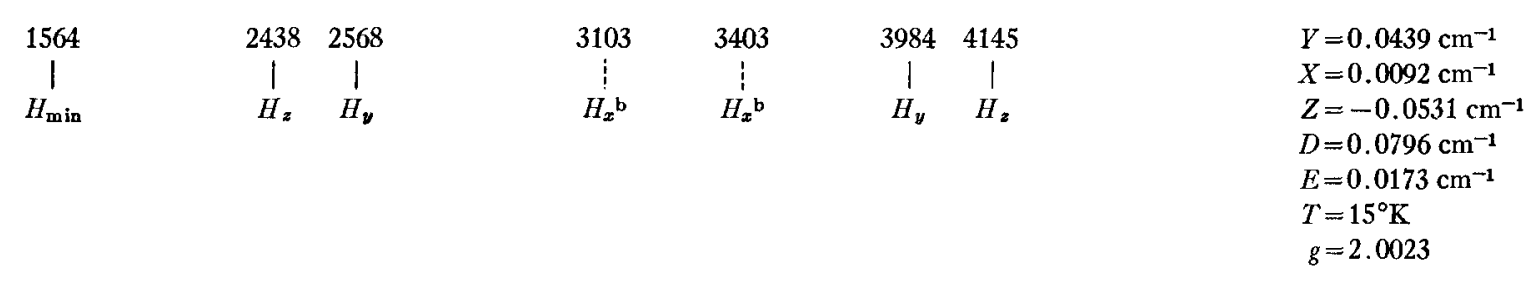

a The approximate center of the broadened signal.

b Dashed lines indicate peaks which were not observed but whose loca- tions were predicted from the spin Hamiltonian. These absorptions were hidden by the free radical signal. large nuclear displacements. As in the case of the free rotational model, if the rate of the pseudorotational motion decreases from a large value or rapid tunneling between equivalent conformations at $77^{\circ} \mathrm{K}$ to a small value at $15^{\circ} \mathrm{K}$ the observed change in the experimental spectra can be accounted for.

The computed peak locations at $15^{\circ} \mathrm{K}$ are in excellent agreement with the experimental values, but the line shapes and relative intensities differ somewhat (see Fig. 3). The differences may be due to some variation of $E$ from site to site, whose average value is the observed $E=0.0173 \mathrm{~cm}^{-1}$, and/or there may be still some intramolecular motion present at $15^{\circ} \mathrm{K}$.

The nature of the pseudorotation in corannulene can only be guessed at presently, but it is believed that Jahn-Teller deformations play an important role. The simple Hückel MO treatment, based on the irreducible representations of the $C_{5 v}$ point group, neglecting electron repulsions, indicates that vibrations of either $e_{1}$ or $e_{2}$ symmetry would be sufficient to remove the fivefold symmetry. Such vibrations would probably exercise their greatest effect on the "soft" 15 -membered outer ring allowing an interconversion among five equivalent conformers. More extensive work is now in progress to understand the nature of the pseudorotation in terms of Jahn-Teller distortions and other vibronic interactions.

The differences between the low-temperature spectra in DMF-EtOH and 2-MeTHF indicate that matrix interactions appear to effect the degree of molecular distortion and the rate of the pseudorotational motion.
In the more polar DMF-EtOH matrix the nonzero value of $E$ is clear evidence of a triplet species frozen into a conformation with less than threefold symmetry. In the less polar 2-MeTHF matrix the linewidth of the features associated with the $X, Y$ magnetic axes narrowed as the temperature was lowered, but no resolution of the $X$ and $Y$ features was achieved at the lowest obtainable temperature. This is indicative of a smaller extent of molecular distortion and a smaller potential barrier for the pseudorotational motion in the less polar 2-MeTHF matrix.

The difference in reported $g$ values at 77 and $15^{\circ} \mathrm{K}$ indicates that spin-orbit interactions may be more significant at the higher temperature. To explain the change in the $g$ value with temperature, a simple model is being investigated. A spin-orbit perturbation could be induced by a pseudorotation which might mix higher-energy orbitally degenerate states with the apparently nondegenerate phosphorescent triplet state. Such a model would predict that spin-orbit contributions would be proportional to vibrational or vibronic interactions which in turn are temperature dependent. Consequently, at lower temperatures, where the molecule is "frozen" in a conformation of less than threefold symmetry, smaller spin-orbit contributions are expected and $g$ would be closer to the free electron value.

\section{ACKNOWLEDGMENTS}

The authors are indebted to Dr. T. M. Dunn, Dr. A. H. Francis, Dr. G. Palmer, and Dr. R. Sands for the use of their equipment during the course of this 
research and finally we should like to thank Dr. R. Lawton for providing us with a sample of corannulene.

* Supported in part by the American Chemical Society Petroleum Research Fund No. 1941-A5.

${ }^{1} \mathrm{~J}$. H. van der Waals, A. M. D. Berghuis, and M. S. de Groot, Mol. Phys. 13, 301 (1967).

${ }_{2}$ M. S. de Groot, I. A. M. Hesselmann and J. H. van der Waals, Mol. Phys. 10, 241 (1965).

${ }^{3}$ M. S. de Groot and J. H. van der Waals, Mol. Phys. 6, 545 (1963)

${ }^{4}$ M. S. de Groot, I. A. M. Hesselman, and J. H. van der Waals, Mol. Phys. 13, 583 (1967).

b M. S. de Groot, I. A. M. Hesselman, and J. H. van der Waals, Mol. Phys. 10, 91 (1965).
${ }^{6} \mathrm{~W}$. Barth and R. Lawton, J. Am. Chem. Soc. 88, 380 (1966).

7 J. Hansen, Ph.D. thesis, University of Michigan, Ann Arbor, Mich. 48104, 1969.

${ }^{8} \mathrm{~J}$. Janata, J. Gendell, C. Ling, W. Barth, L. Backes, H. Mark, and R. Lawton, J. Am. Chem. Soc. 89, 3056 (1967).

${ }^{\circ} \mathrm{D}, \mathrm{H}$. E. Ingram, Free Radicals as Studied by ESR (Butterworth, London, 1958).

${ }^{10} \mathrm{~J}$. Lhoste, A. Haug, and M. Ptak, J. Chem. Phys. 44, 654 (1966).

${ }_{11}$ E. Wasserman, L. C. Synder, and W. A. Yager, J. Chem. Phys. 41, 1763 (1964).

${ }^{12}$ C. A. Hutchison Jr. and B. W. Mangum, J. Chem. Phys. 34, 908 (1961).

${ }_{13} \mathrm{P}$. Kottis and R. Lefebvre, J. Chem. Phys. 41, 379 (1964).

${ }^{14}$ A. W. Hornig and J. S. Hyde, Mol. Phys. 6, 33 (1963).

\title{
Observed Intensity and Speed Distributions of Thermal-Energy Argon Atoms Scattered from the (111) Face of Silver*
}

\author{
M. N. Bishara $\dagger$ and S. S. Fisher \\ Department of Aerospace Engineering and Engineering Physics, University of Virginia, Charlottesville, Virginia 22901
}

(Received 6 November 1969)

\begin{abstract}
The scattering of thermal-energy monatomic gases from metal surfaces is studied using the Ar-Ag system. The argon atoms are incident to the surface in the form of nearly monoenergetic beams from a gas-dynamic nozzle source. The target surface is the polished (111) face of a monocrystalline silver disk. In studying this interaction, essentially complete maps of the relative intensities and speeds of the scattered atoms are obtained using a time-of-flight method. The argon beams have energies ranging from 0.06 to $0.17 \mathrm{eV}$ and are incident at angles varying from $40^{\circ}$ to $60^{\circ}$ from the target normal. The surface temperature ranges from 550 to $790^{\circ} \mathrm{K}$. Due to surface roughness, approximately $80 \%$ of the incident atoms are scattered diffusely while the remainder are scattered into a crest-shaped lobe centered subspecularly within the plane of incidence. To a first approximation, it is shown that the speed distributions of the diffusely scattered components are independent of scattering angle and that they are nearly Maxwellian in form and exhibit mean speeds corresponding to high thermal accommodation to the surface. The lobularly scattered atoms show a clear tendency to conserve their momentum tangential to the surface. Moreover, it appears that the characteristics of the two component beams are relatively independent of the fraction scattered into each. General properties of the scattered beams are discussed and comparisons with applicable studies are offered.
\end{abstract}

\section{INTRODUCTION}

Argon atomic beams have been scattered from the polished (111) face of silver crystals and both the intensity and the speed distribution of the scattered atoms have been measured over a network of directions covering a large fraction of the solid angle subtended by the scattered beams. The technique used is identical to that employed in a previously published study ${ }^{1}$ in that nearly monoenergetic, aerodynamicsource atomic beams, along with time-of-flight detection methods, are employed. The principal differences between the previous study and the present one are (a) that observations are no longer restricted to the plane of incidence alone, and (b) that the target is a monocrystalline metal. The gas-surface combination employed here was chosen because there are many available experimental data in the literature ${ }^{2-4}$ with which to compare at least part of the results (primarily the intensity distributions of the scattered atoms) and because this combination has been utilized in demonstrating the characteristics of several available theories. ${ }^{5-8}$

The speed measurements made possible by the timeof-flight technique have added a new dimension to the interpretability of the results. The interaction "strength" can now be estimated, as a function of scattering direction, in terms of the extent of randomization of the speed distribution of the nearly monoenergetic incident beam. In addition, mean speeds and mean-square speeds can be calculated as functions of scattering angle thus making it possible to determine and assess momentum and energy exchange between the gas and the surface.

It is now widely accepted that an appropriate model for the distribution of intensity in the scattering of inert, thermal-energy gases from heated metal surfaces is one for which some sizable fraction of the molecules is scattered into a lobe centered within the plane of incidence. This lobe, whose intensity maxi- 